\title{
A novel predictive optimization scheme for energy-efficient reliable operation of a sensor in dynamic scenarios
}

\author{
Jayasri B. S., G. Raghavendra Rao \\ Department of Computer Science, National Institue of Engineering, India
}

\begin{tabular}{|c|c|}
\hline Article Info & ABSTRACT \\
\hline $\begin{array}{l}\text { Article history: } \\
\text { Received Jun 15, } 2019 \\
\text { Revised Aug 20, } 2019 \\
\text { Accepted Aug 29, } 2019\end{array}$ & $\begin{array}{l}\text { Wireless Sensor Network (WSN) has been studied for more than a decades } \\
\text { that resulted in evolution of the significant applications towards assisting in } \\
\text { sensing physical information from human inaccesible area. It was also } \\
\text { observed from existing sysem that energy attribute is the root cause of } \\
\text { majority of the problems associated with WSN that also gives rise to various } \\
\text { operational reliability issue. Therefore, the prime goal of the proposed study }\end{array}$ \\
\hline $\begin{array}{l}\text { Keywords: } \\
\text { Energy } \\
\text { Optimization } \\
\text { Predictive } \\
\text { Reliability } \\
\text { Wireless sensor network }\end{array}$ & $\begin{array}{l}\text { is to present a novel predictive optimization approach of data fusion in order } \\
\text { to jointly address the problems associated with energy efficiency and reliable } \\
\text { operation of sensor nodes in WSN. An analytical research approach is carried } \\
\text { out in order to ensure that a time-based synchronization scheme contributes } \\
\text { to offer an evolutionary approach towards significant energy optimization. } \\
\text { A simulation-based benchmarking analysis is carried out to find that } \\
\text { proposed system offers good energy-efficient performance in comparison to } \\
\text { existing approaches. }\end{array}$ \\
\hline & $\begin{array}{r}\text { Copyright } @ 2020 \text { Institute of Advanced Engineering and Science. } \\
\text { All rights reserved. }\end{array}$ \\
\hline \multicolumn{2}{|l|}{ Corresponding Author: } \\
\hline $\begin{array}{l}\text { Jayasri B. S., } \\
\text { Department of Computer Science, } \\
\text { National Institute of Engineering, } \\
\text { Mysore, India. } \\
\text { Email: jayasriphd14ni@gmail.com }\end{array}$ & \\
\hline
\end{tabular}

\section{INTRODUCTION}

Wireless Sensor Network (WSN) is one of the most adopted networking technologies that have found its identity from the arena of research to commercial products and services [1]. At present, there are numbers of organization developing sensor nodes with different capabilities of sensing different physical attributes. Although, sensor motes are capable of extracting different information from surrounding but it's computational processing is carried out by a firmware called as node and both together combined to be called as a sensor node. After the sensors are dispersed in human inaccessible area, they have to undergo a process of clustering in order to perform an organization process of acquiring and forwarding the data to the base station. This process is called as data aggregation and there are various literatures e.g. [2-4] that have addressed various problems associated with. However, very often a process called as data fusion is overlooked that is the initial processing of the data within a sensor. Unfortunately, there is no much in-depth investigation towards the process of data fusion except some of the reported literatures on data fusion e.g. [5-7]. It will mean that inspite of significant difference between data fusion and data aggregation process, emphasis is more on latter one.

There are various reason behind it viz. a) working on data fusion problem will require the protocol to be designed from local level of raw data processing, which becomes quite a complex process in heterogeneous networking scenarios, b) effectivity of applying different data fusion approaches over same or different clustering is yet unknown, c) assessment of scalability problems considering dynamic networks during data fusion is quite a computationally challenging problems. A closer look into above mentioned problem will show that owing to lesser degree of research towards data fusion, it will significant give rise to 
energy drainage problems among the sensor nodes in WSN. Owing to less number of research towards exploring the impact of data fusion process over energy efficiency except some of them e.g. [8, 9], there is a significant need to carry out investigation towards this direction. It is because if the energy problems are not associated with respect to data fusion process, the nodes will start shooting reliability problems too.

By reliability problem, it will mean that amount of network lifetime that the new protocol is capable of ensuring irrespective of any uncertain traffic condition in WSN. Reliability factor of energy-efficient node operation is highly essential for some of the sensor applications e.g. healthcare monitoring, natural calamities monitoring, chemical plant monitoring, etc., where uncertainty of even occurances causes massive drainage of energy and this is reflected by unequal network lifetime thereby affecting the reliability factor. Therefore, there is a potential need of a novel approach that emphasizes only on improving data fusion process in order to offer good energy saving as well as maintain greater degree of reliability too. The proposed paper presents a unique framework design where a simple predictive approach of optimization is carried out using a novel evolutionary technique completely different than any existing system.

The target of this study is to offer reliable sensory performance. The Section 1.2 briefs about related work as a background associated with energy-based solution as well as reliability-based solution in WSN. It also defines exclusive research problems in the consecutive Section 1.2 The fundamental concept about proposed research methodologies to solve the reported research problem is discusses in Section 1.3 that is further followed by Algorithm design in Section 2. Section 3 discusses about accomplished outcome whereas Section 4 briefs about final conclusion on the proposed study

The reviews of existing approaches towards energy efficiencies in WSN can be referred from our past investigation [10] whereas we update more on existing approaches in relation with reliability problems in this paper. Most recently study towards energy and reliability problems has been addressed by Alshaheen and Takruri-Rizk [11] using network coding approach. Study towards reliability problems was also addressed in the work of Duan et al. [12] by amending software defined network for addressing node failures issues owing to energy consumption. Qadori et al. [13] have used fuzzy-approach along with consideration of mobile agents in order to conserve energy consumption. Zhang et al. [14] have presented an adaptive algorithm for ensuring cost effective exchange of control message over dynamic network condition for ensuring good reliable operation of sensor. The problems of node reliability are also addressed by Zhu et al. [15] in the context of physical layer security.

The work carried out by Fernandes et al. [16] have presented a scheme to minimize transmission power consumption using received signal strength for addressing overhead problems. Adoption of gametheory has been also connected for solving scheduling problems for energy saving in WSN as witnessed in the work of Kang et al. [17]. An empirical-modelling based approach was presented by Feng and Dong [18] in order to investigate reliability issue. The work carried out by Liu et al. [19] have discussed about usage of non-convex optimization problem for solving allocation of power issues in body area network while performing energy harvesting. Usage of context-based sensing mechanism was also proven to affect energy saving as reported in the work of Prabha et al. [20].

Samantha and Misra [21] have emphasized on delay problems as well as minimization of cost factor involved in network management considering opportunistic network optimization. Wang et al. [22] have presented a hierarchical-based approach along with usage of scheduling policies for ensuring better energy saving for distributed operations among the sensors. Reliability issues were also investigated with respect to fog-computing in order to better establish relationship with sensor devices and cloud environment. Such work has been presented by Wang et al. [23] that perform computation of trust in order to prove reliability of the sensor. A combined study of energy, cost, and reliability has been presented by Zonouz et al. [24] where the authors have considered heterogeneous sensory application using routing protocol deployment to ensure reliability of the path. Wolf et al. [25] have discussed about usage of data correlation-based approach for ensuring better policy for power optimization in WSN.

Literatures have also witnessed clustering-approach for performing energy-optimized operation in WSN. The work of Naeem et al. [26] has presented a cooperative clustering approach with multiple-input multiple-output channels for enhancing the network lifetime. Lei et al. [27] have presented a study where the reliability as well as optimization problems were associated with the energy harvesting. The technique has used markov decision approach as well as stochastic learning approach for leveraging energy allocation process. Liu et al. [28] have presented a technique where throughput is targeted for maximization using non-convex optimization process as well as threshold defined on the basis of signal-to-noise ratio factor. Wu et al. [29] have presented a unique data forwarding process using compressive sensing and various other energy and frequency related factors for ensuring energy efficiency in heterogenous sensory application.

Nearly, similar approach was also presented by Campobello et al. [30] that has utilized a unique remainder theorem for enhancing energy saving features as well as reliability problems among sensory applications. Therefore, there are various reported works at present to disuss about energy and reliability 
problems in WSN. Chisab [31] a modified technique is presented to reduce energy uses per data bit in a connection. Manjunath and Rao [32] have demonstrate a method of security in the context of WSN by presenting two kinds of sensor node deployed with various ablities. The above-mentioned studies have significant advantage as well as technical pitfalls too that are outlined in next section.

The significant research problems are as follows:

a. There is a smaller number of cost-effective optimization process for addressing energy and reliability problem in sensory application in existing studies.

b. The existing studies lack an effective energy modeling with respect to data fusion process which is prime operation to initiate data aggregation proces in WSN.

c. There are less benchmarked approaches and techniques to be reported for a good balance between energy and communication performance.

d. Study towards computational complexity associated with the usage of predictive approaches is ignored and hence further insight toward cost effective architecture designing is halted.

Therefore, the problem statement of the proposed study can be stated as "Constructing a costeffective, simple, and yet robust predictive optimization algorithm for ensuring energy and reliability issues in sensory application in dynamic scenario is quite a challenging task". The next section outlines solutions.

The proposed study is continuation of our prior works [33-35] where the energy consumpsion problems while obtaining reliable data fusion process are addressed to a significant aspect. However, the proposed study also aims to design a novel computational framework well capable of performing cost effective optimization process towards achieving better solution in terms of energy efficiency and reliable data fusion and communication in a large scale WSN. The study basically formulated an analytical methodology enabled computational modelling to address the energy and reliability problems associated with conventional sensory applications. It basically designed a framework which assesses TDMA-CSMA based time-scheduling approach in order to obtain a good balance between energy and communication performance of a WSN. The designed methodology of the proposed system is highlighted in Figure 1.

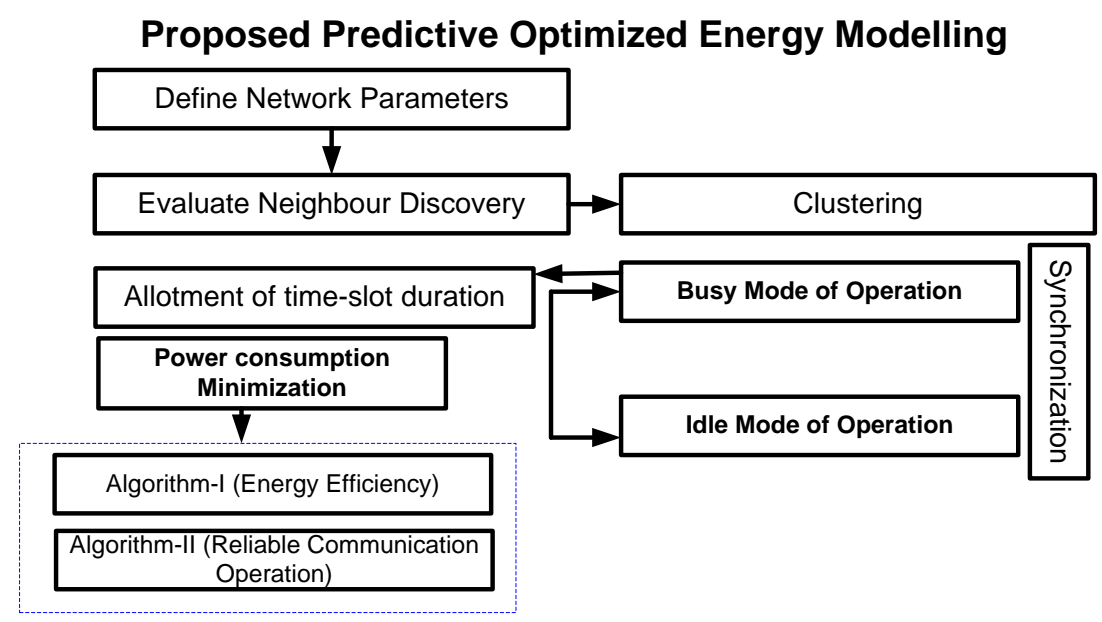

Figure 1. Implemented schema of proposed system

The Figure 1 represents the design methodology adopted into proposed energy efficient and reliable data delivery model for a large scale WSN. The analytical desings adopted into the proposed framework design are further implemented in the form of two different algorithms which are simulated to ensure optimized and energy efficient time scheduling for reliable communication in a WSN. The proposed system evaluates a clustering paradigm prior to data fusion which makes core aggregator mote/Cluster heads busier with heavy computation. Thereby the proposed study emphasized on reducing the energy consumpsion during the communication and data transmission phase where TDMA-CSMA scheduling applies two different mode of operation for reliable channel access where binding attrbutes utilizes four different time slots very effectively with a parallal and joint routing-based strategy. The proposed study evaluates power consumpsion minimization strategy with respect to two different algorithms which are extensively discussed in the next section. Finally, the study performs a comparative analysis in order to validate theexperimental outcomes obtained after simulating the proposed schema in a numerical computing environment. 


\section{IMPLEMENTATION}

In this section, the study exclusively demonstrates the implementation strategy of the proposed computational framework for performing efficient data fusion in a data-driven WSN through optimizing the performance efficiency of packet delivery paradigm in terms of energy and reliability. In order to do so, the study uses a novel decision-oriented data delivery schema with predictive coding. However, the study attempted to design a novel framework well capable of performing data fusion by jointly addressing the problem of energy efficiency and issues of reliability in a WSN from both functional and operational aspects. It mostly puts emphasize towards classifying the data packets with the deployment of cost-effective Time Division Multiple Access (TDMA) and Carrier Sense Multiple Access (CSMA) subjected to decisional and efficient energy modelling.

The proposed system performs data fusion prior to data aggregation when aggregator nodes act as cluster head $\left(\mathrm{C}_{\mathrm{h}}\right)$ while operating on diffent sensor node $(\mathrm{SN})$ driven clusters. The process thereby mainly follows two different types of communication phases such as i) communication between node to $C_{h}$ where data fusion take place and ii) communication between each $C_{h}$ to core aggregator mote, data aggregation is performed. The prime emphasize has been given towards energy consumpsion optimization by performing reliable sensory operations with synchronized packet delivery paradigm with randomized and organized access mode of processes. The data delivery cycle has been conceptualized in a way where a time slot is defined for transmission operations, also referred as temporal interval $\left(t_{\mathrm{i}}\right)$. $t_{\mathrm{i}}$ basically consists of two different types of mode of operations a) busy mode and b) idle mode respectively. The system also considers slot of busy duration ratio $\left(r_{\mathrm{c}}\right)$ in which a pair of nodes here node $n_{\mathrm{i}, \mathrm{j}}$ and node $\mathrm{n}_{\mathrm{j}, \mathrm{i}}$ (where $\mathrm{i}, \mathrm{j} \in Z^{+}$) might be a member node and a particular cluster where cluster head $\mathrm{C}_{\mathrm{h}}$ forms a communication channel for transmission and reception and explicitly communicate with each other prior to performing data fusion.

The proposed framework basically classifies the sensed data packets according to their priority and arranage their priority sequence by maintaining an appropriate job queue $\mathrm{J}_{\mathrm{Q}}$. The source node sets 1 bit priority value to each data packet which is basically appended in application layer. Energy optimization by addressing the reliable operation of communication with optimized time synchronized fixed leangth of slots $t_{\mathrm{i}}$ in a WSN has not yet obtained its full-proof solutions, thereby the proposed study has extended its research effort towards optimized decision making to obtain maximum possible energy efficient and reliable data trasnsmission prior perforimng fusion in core aggregator nodes. The source nodes arrange all the sensed data according to their respective degree of priority. The communication process is organized with respect to fixed number of time slots where in busy mode of operation fixed number of time slots in which busy mode of operation enable synchronization of data packets during transmission phase followed by a request-to-send (RTS) and clear-to-send (CTS) control signals. The busy mode operates on randomized access schema of combined TDMA-CSMA for the ease of efficient usage of bandwidth and channel access. On the other hand, during idle period the syatem operates on oraganized access mode where data and acknowledgement signals get transmitted from sender to receiver nodes. The synchronization among data packet transmission in this regard take place within the same cluster using a control packet $\lambda$.

\subsection{Proposed numerical simulation study}

This section discusses about how the proposed simulation environment designed to assess the performance of the novel predictive optimization scheme and its significant impact on achiving energy efficient reliable operation in a data driven WSN. The prime focus here lay upon data fusion where most of the energy of sensor nodes get consumed and affects the performance of the system in terms of energy and other QoS aspects.

\subsubsection{Defining different network parameters}

The proposed system initially defined different network parameters during computational modelling and numerical simulation procedure. There are various network parameters considered while modelling the WSN environment such as deployment area $(A)$, number of sensor nodes $(\eta)$ to be deployed followed by deployment of the sink node by locating its coordinates $\left(x_{\mathrm{BS}}, y B S\right)$. However, the proposed system enabled its computation and simulation on a 64-bit operating system driven with x64-based processor. After successful deployment of sensor nodes within a particular area the process computes the distance between one node and its neighbor and determine communication radius with respect to data packet transmission duration $\left(T_{\mathrm{pkt}}\right)$ with temporal interval. The process continues and checks the neighbours of each node by incrementing communication radius $\left(\mathrm{C}_{\mathrm{r}}\right)$. The proposed system considers $r_{\mathrm{c}}$ within a range (0.05-0.5) during implementation. 


\subsubsection{Proposed routing schema}

After successful completion of node deployment stage, the proposed system basically initiates neighbor discovery phase where virtual clustering plays a crucial role towards synchronizing the data packet transmission from sensor node to $\mathrm{C}_{\mathrm{h}}$. It is basically defined with a time slot divided into busy and idle mode of operations. When a sensor node begins with its activation and initiates its operational stage at that point it listens and do wait for route request packets during its busy mode. A perticluar time slot $\mathrm{t}_{\mathrm{A}}$ is defined which clearly states till a certain period an active node will listen after that it will broadcast an advertisement packet $P_{\mathrm{ADV}}$ with a perticluar time slot frame. The advertisement broadcasted packet $P_{\mathrm{ADV}}$ contains duration time until the next packet starts its transmission phase. During busy of operation nodes broadcast their advertisement packet $P_{\mathrm{ADV}}$ once in a while.

Every node connected to the network follows the packet transmitted from one node and its duration scheduled in each frame. If a node with a distinct pattern of duration schedule listens to a $P_{\mathrm{ADV}}$ with different duration schedule, then that node participates in communication ensuring its authenticity and reliability. The proposed study adopted the clustering phase in a way where nodes having distinct pattern of duration schedule formulate a cluster with same schedule for network operation i.e. nodes belong to a particular cluster will follow same duration scheduled for operation. During neighbor discovery and clustering operations each sensor node creates a matrix containing its one hop and two neighbor lists and assigned with a particular id. The checks if distance between two nodes is less than the commincation radius $R$ of each node then it formulates communication link prior to clustering. The communication duration is formulated with fixed length slots which includes busy period and sleep period. During busy period the synchronization happens with respect to RTS/CTS in an integral CSMA/CA paradigm. The idle period is set in a way where maximum possible data with ACK packets are allowed to be transmitted. The proposed system evaluates one-hop and two-hop neighbor discovery phase with allotement of time-slot duration within a frame.

\subsubsection{Allotment of time-slot duration}

The allotment of time slot duration for different sensor node has been initiated considering a state transition-based scheme where in the idle mode of operation the duration should be long enough so that node can transmit maximum possible data with ACK. The study also formulated a priority-based scheduling of slots where data packets comprising significant data bits get higher priority and also subjected to claim for any slot of time duration for its operation. If no such data is present in the network operation, then the proposed system allot slot of frame to the proprietor of a particular time interval. The proposed system perfoms assignment of slot/frame based on $v$ which is a modulo value computed for two-hop neighbours.

\subsubsection{Power consumpsion minimization phase}

When a sensor is sitting idle at that stage it disables its radio communication and wait according to its predefined duration recommended by busy duration ratio. Once the duration gets over then it switches back to its active mode of operation by intitating its radio and start listening to the channel medium. Incase if it finds any data which is needed to be transmitted or to be received, thereby it starts operating on CSMA/CA else on time out it again move back to its idle mode. If a sender node successfully claims to win argument then both sender and receiver goes to Tx/Rx state and on the completion of successful communication sender and receiver go to idle state. Nodes who fail to win argument directly go to idle mode. It also enables a collision free data transmission with simultaneous IEEE 802.11 scheme.

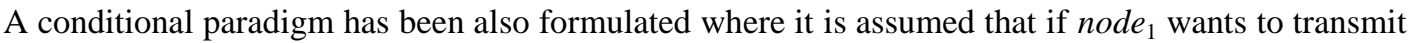
any data packet to node $_{2}$, then it will initially transmit an RTS to node 2 . As node 3 lies within the communication range of node $e_{1}$ thus it can overhear the RTS and convey a decision making scnerio that the transmission medium/communication channel is engaged in active/busy mode of operations. Now, another condition where node $_{3}$ has to transmit its data to node $_{4}$, therefore node $e_{3}$ at this point will not send any data as it knows the communication channel is busy but on the other hand it can also be seen in the Figure 2 that transmitting data from node $_{3}$ to node $_{4}$ also will not affect the communication between node $_{1}$ and node $_{2}$. It should be noted that the proposed system also considered a scnenrio where it is stated that if a node lies within the transmission or communication range of a sender node then it can receive the packet and can decode it but if a node is located within the carrier sensing zone of a sender node then only it can sense and hear the transmission being performed within a particular busy/idle time of interval.

The proposed system also deployed a schema to adjust transmission power during busy mode of operations where unique pattern of topology formation with optimized predictive coding increses the set of communication links and also reduced the communication delay with reliable and simultaneous data transmission. The proposed system considers formulating two different types of algorithms where the first algorithm is designed for cost effective predictive modelling of energy efficient communication process for identifying optimized link formation prior to data fusion process which also reduces the collision in dynamic

A novel predictive optimization scheme for energy-efficient reliable operation of a sensor ... (Jayasri B. S.) 
traffic condition. The second algorithmic approach formulated with an analytical notion to ensure reliable set of communication operations in CSMA schema with transmission power adjustment during data fusion process between node to its core aggregator node or $\mathrm{C}_{\mathrm{h}}$.

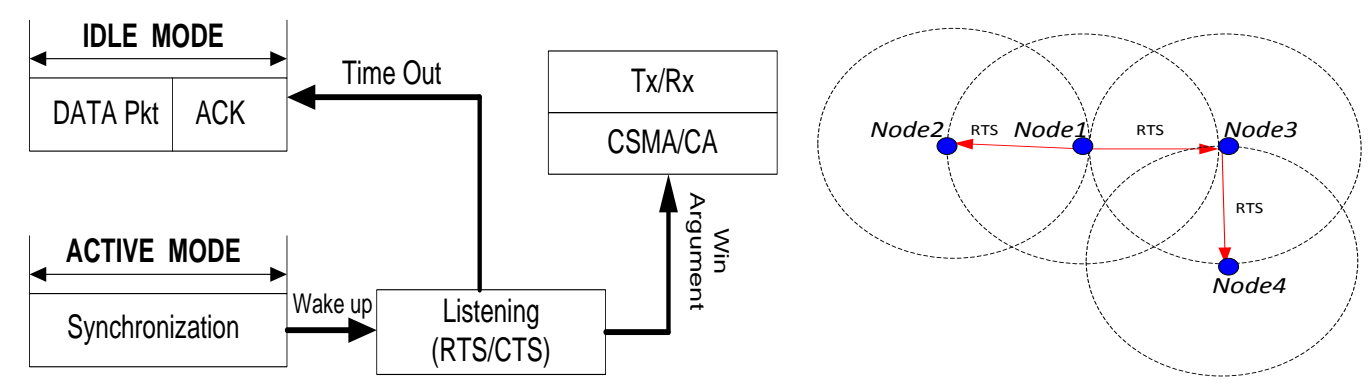

Figure 2. Proposed energy efficient data collision avoidance schema

Algorthm 1: a novel predictive and efficient energy modelling

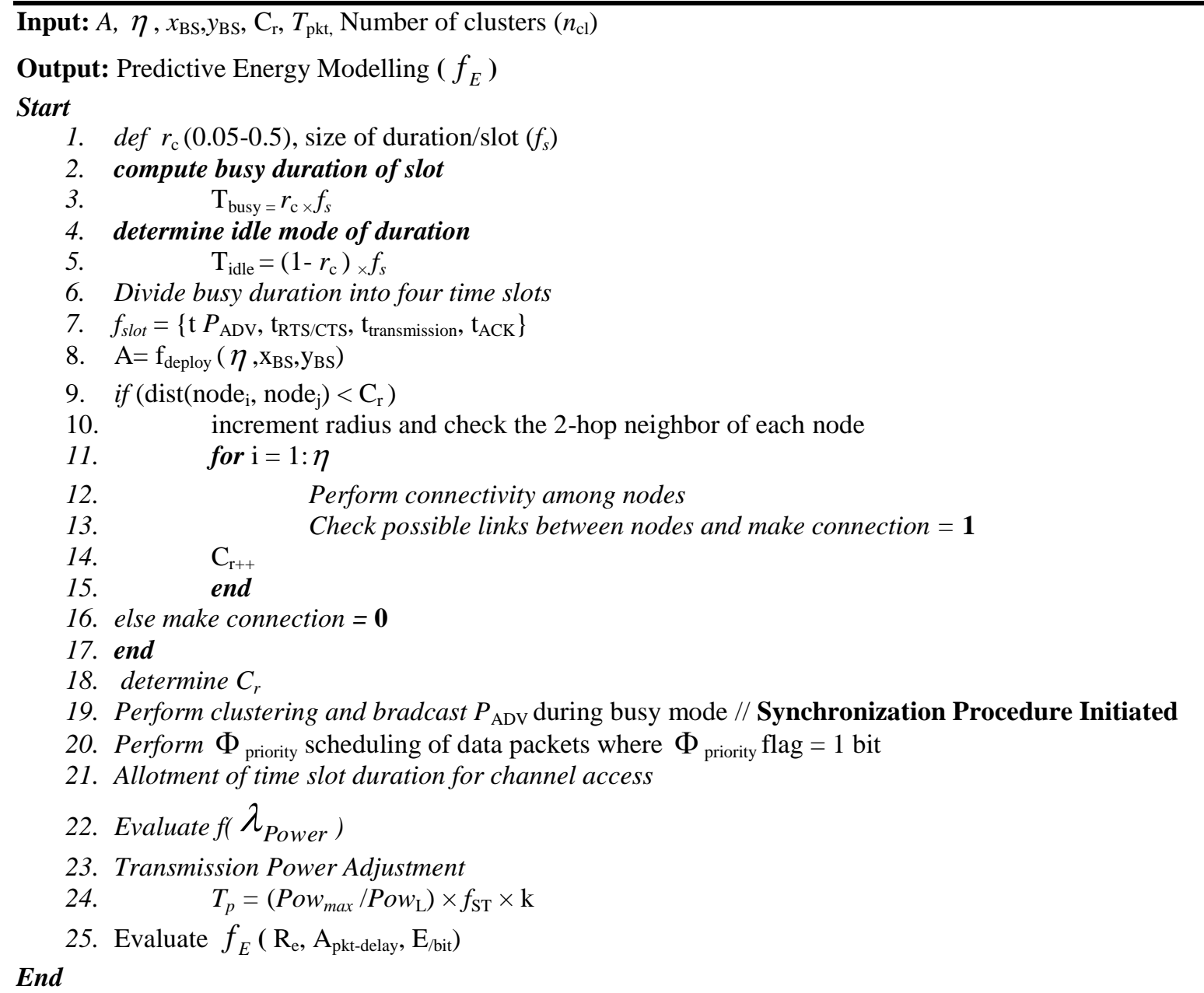

The algorithm 1 shows how the proposed optimized modelling evaluated its novel power consumpsion minimization procedure by synchronizing data packet arrival duration with effective link and broadcast shcehduling schema. The transmission power adjustment prior to the data fusion process is evaluated by simulating the eq. highlighted in (Line-24). The system is modelling in a way where prior to fusion process when each sensor node wants to transmit data to its neighbor at that point it can adjust its transmission power to optimize the energy consumpsion. The proposed system thereby allow transmission of 
RTS/CTS packets with maximum power $P o w_{\max }$ in both transmitter and receiver side as well. When a sender node receives a CTS packet at that time it computes $T_{p \text { with }}$ respect to the power level received Pow $_{\mathrm{L}} . f_{\mathrm{ST}}$, k in above (Line-24) denotes minimum necessary signal strength and constant respectively. These all attributes are computed further prior broadcasting ACK messages with data bits during idle mode of operation. Finally the proposed system computes Evaluate residual energy $\left(\mathrm{R}_{\mathrm{e}}\right)$, Average Packet Delay $\left(\mathrm{A}_{\mathrm{pkt} \text {-delay }}\right.$, Energy Consumpsion/bit $\left(\mathrm{E}_{/ \mathrm{bit}}\right)$ in (Line-25).

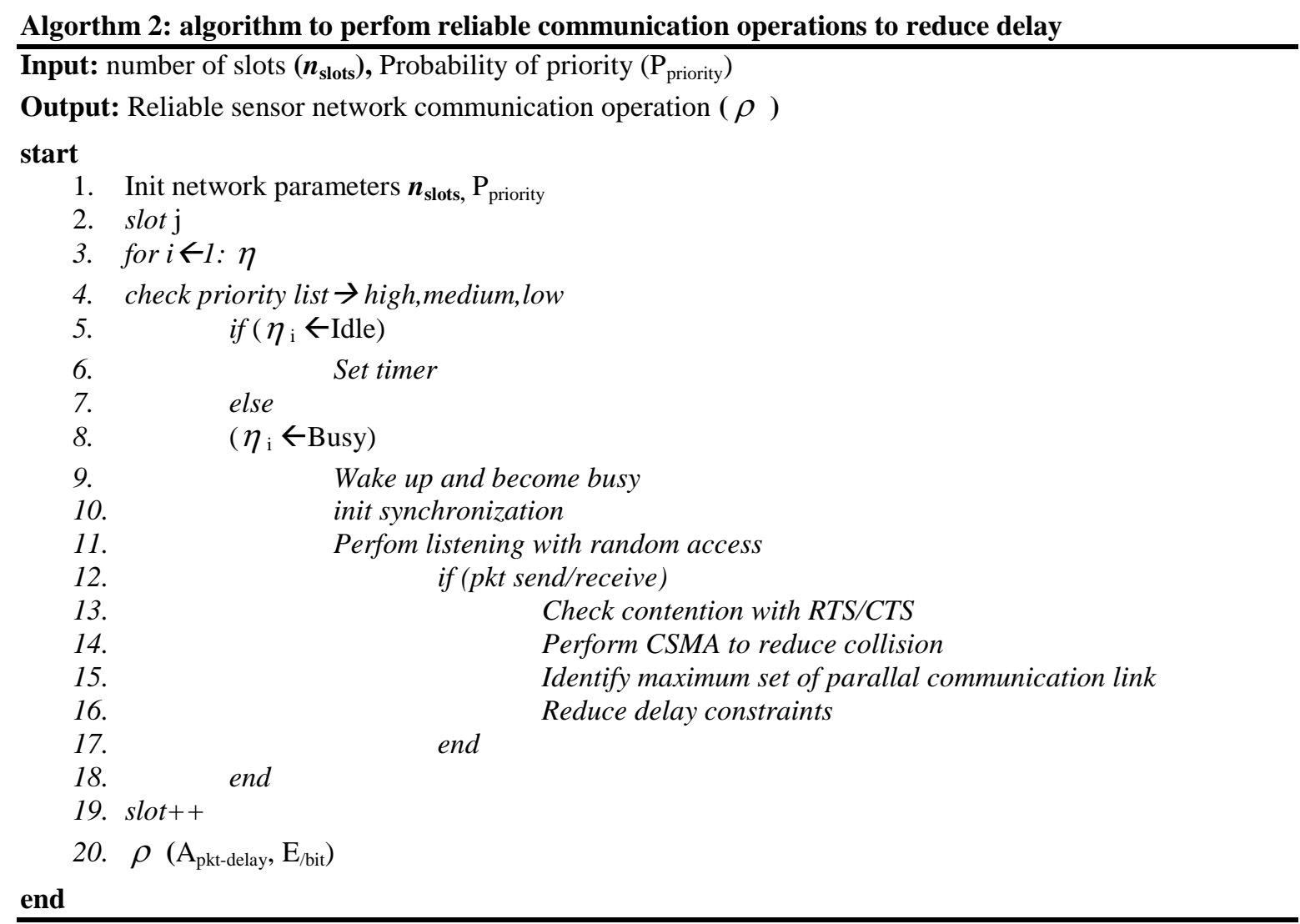

The algorithm 2 also demonstrates how the proposed system ensures optimized reliable communication by reducing the delay which arises due to collision and other network constraints. The proposed algorithm subjected to perform synchronized communication operation during the data fusion phase where the clustering and data communication with respect to parallal transmission using CSMA scheme ensured energy reduction. The idea of state transition phase for each node where one node will periodically wake to see any other node wants to communicate or node along with two different modes of operations such as busy and idle makes the system more accountable towards utilizing the energywith very reliable manner.

The proposed system is also designed in a way where a set of nodes belong to a particular cluster will follow the same schedule in every instance of time. The proposed system also applies a packet transmission scheme where nodes belong to a particular $\mathrm{C}_{\mathrm{h}}$ like node $_{1}$, node $_{2}$, node node $_{4}$ will be adjacent to each other as they will lie within each others transmission range and will follow the same schedule of operation during communication from node to cluster head. The proposed study divided the overall busy duration ratio into four different slots such as $\mathrm{j}, \mathrm{j}+1, \mathrm{j}+2, \mathrm{j}+3$. The first slot is defined for $P_{\mathrm{ADV}}$ which performs synchronization with CTS/RTS packets. The next portion is idle mode of operation when the data transmission takes place in between two consequtive nodes. While data transmission take place between two adjacent nodes at that pont of time nodes lie within their communication range also listen to the channel if there is any incoming data waiting for them at queue. The traffic management operation with respect to effective utilization of channel access on priority makes the proposed system more reliable from energy efficiency aspects.

The next segment of the study demonstrates the outcome being accomplished after simulating the proposed system on a numerical computing environment. The study also further assesses a comparative performance analysis to validate the authenticity and outcome of the proposed algorithms. The outcome of the proposed study further compared with two of the existing baselines proposed by Luo et al. [36] and

A novel predictive optimization scheme for energy-efficient reliable operation of a sensor ... (Jayasri B. S.) 
Zhang et al. [37]. However, the comparative performance analysis further conveyed that the proposed approach of energy efficient predictive schema for TDMA-CSMA accomplises optimal outcomes with respect to different performance parameters considered.

\section{RESULT ANALYSIS}

This section of the proposed study represents the outcome accomplished after simulating the above two algorithms in a numerical computing environment. The outcomes obtained are further compared with the existing baselines (i.e. the work carried out by Luo et al. [36] and Zhang et al. [37]). The Figure 3 exhbits the results obtained for remaining energy in the case of proposed system for (2-10) number of clusters and 10 round of simulation and it is quite clear that the the curve orientation in proposed approach is quite higher as compared to the Luo [36] and Zhang [37] approach. Thereby, deployment of energy efficient clustering during data fusion along with CSMA-TDMA based time slot scheduling certainly maximizes the network life time of the deployed WSN.

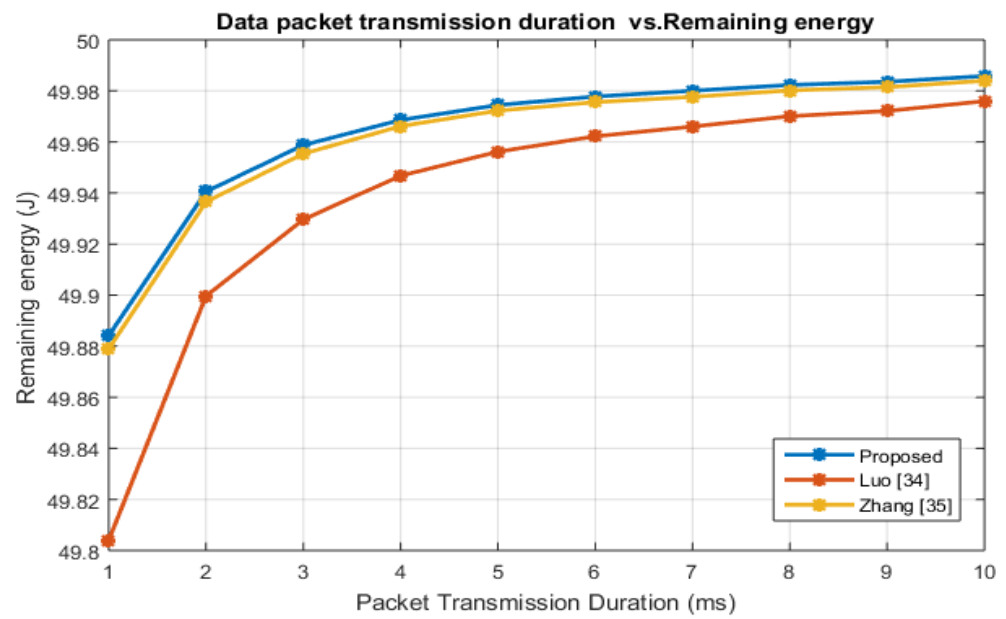

Figure 3. Comparative analysis (remaining energy vs data packet transmission duration)

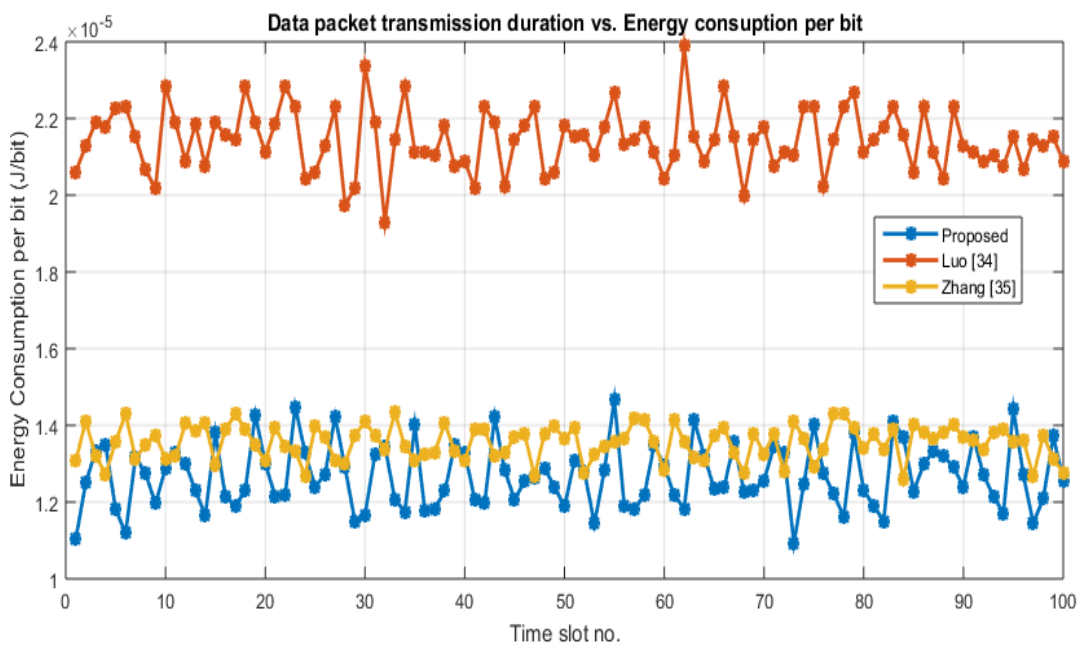

Figure 4. Comparative analysis (time slot no vs energy consumpsion j/bit)

The proposed two algorithms are simulated with respect to priority-based scheduling which utilizes maximum number of time slots synchronized with communication medium in order to obtain maximal 
number of communication links. The remaining energy is found quite higher in the case of proposed system even though simulating it with higher number of periodic intervals.

The Figure 4 also exhibits that the proposed two algorithms also achieves reliable communication operations to reduce the delay constraints which has a significant impact on energy consumpsion optimization. The above Figure 4 also shows that energy consumpsion per bit in every instance of increasing time slots is higher in the case of Luo [36] where, Proposed approach and Zhang [37] approach accomplishes optimal outcome as they both utilized predictive node scheduling in a dynamic environment where nodes are actually performs decision making during scheduling and data fusion process. However, as our proposed system excels better outcomes with respect to different attributes thus the study ensures its uniformity towards achiving reliable communication scenario during data fusion process in a WSN.

\section{CONCLUSION}

As the recent advancement in the field of wireless communication, confines various operational resources in a WSN, therefore balancing the trade-off between energy consumpsion and reliable communication operation has become a crucial challenge for the researchers. The proposed study thereby addressed the aforementioned issues of WSN during data fusion and formulated a novel predictive energy modelling to ensure reliable communication with minimal usage of node power. Finally, a comparative performance analysis has exhibited that our proposed scheme outperforms the existing baselines in terms of energy efficiency and reliability. The reliability factor here ensured cost effective communication to reduce delay constraints.

\section{REFERENCES}

[1] Y. Peng, et al., "Enhancing Energy Efficiency via Cooperative MIMO in Wireless Sensor Networks: State of the Art and Future Research Directions," IEEE Communications Magazine, vol. 55, pp. 47-53, 2017.

[2] M. Bagaa, et al., "Data Aggregation Scheduling Algorithms in Wireless Sensor Networks: Solutions and Challenges," IEEE Communications Surveys \& Tutorials, vol. 16, pp. 1339-1368, 2014.

[3] J. Xu, et al., "A survey on the privacy-preserving data aggregation in wireless sensor networks," China Communications, vol. 12, pp. 162-180, 2015.

[4] D. Wong and M. H. Wong, "Fast and Simultaneous Data Aggregation Over Multiple Regions in Wireless Sensor Networks," IEEE Transactions on Systems, Man, and Cybernetics, Part C (Applications and Reviews), vol. 41, pp. 333-343, 2011

[5] X. Jiang, et al., "Data Fusion-Based Multi-Object Tracking for Unconstrained Visual Sensor Networks," IEEE Access, vol. 6, pp. 13716-13728, 2018.

[6] Z. J. Zhang, et al., "A green data transmission mechanism for wireless multimedia sensor networks using information fusion," IEEE Wireless Communications, vol. 21, pp. 14-19, 2014.

[7] E. Baccarelli, et al., "Green multimedia wireless sensor networks: distributed intelligent data fusion, in-network processing, and optimized resource management," IEEE Wireless Communications, vol. 21, pp. 20-26, 2014.

[8] X. Chen and Y. Li, "Optimal Energy Allocation to Maximize Network Utility of Wireless Sensor Networks Based on Data Fusion," 2015 Sixth International Conference on Intelligent Systems Design and Engineering Applications (ISDEA), Guiyang, pp. 551-554, 2015.

[9] H. Kalantarian, et al., "Multimodal energy expenditure calculation for pervasive health: A data fusion model using wearable sensors," 2013 IEEE International Conference on Pervasive Computing and Communications Workshops (PERCOM Workshops), San Diego, CA, pp. 676-681, 2013.

[10] Jayashri B. S and G. R. Rao, "Reviewing the research paradigm of techniques used in data fusion in WSN," 2015 International Conference on Computing and Communications Technologies (ICCCT), Chennai, pp. 83-88, 2015.

[11] H. Alshaheen and H. Takruri-Rizk, "Energy Saving and Reliability for Wireless Body Sensor Networks (WBSN)," IEEE Access, vol. 6, pp. 16678-16695, 2018.

[12] Y. Duan, et al., "A methodology for reliability of WSN based on software defined network in adaptive industrial environment," IEEE/CAA Journal of Automatica Sinica, vol. 5, pp. 74-82, 2018.

[13] H. Q. Qadori, et al., "FuMAM: Fuzzy-Based Mobile Agent Migration Approach for Data Gathering in Wireless Sensor Networks," IEEE Access, vol. 6, pp. 15643-15652, 2018.

[14] Y. Zhang, et al., "A Blind Adaptive Tuning Algorithm for Reliable and Energy-Efficient Communication in IEEE 802.15.4 Networks,” IEEE Transactions on Vehicular Technology, vol. 66, pp. 8605-8609, 2017.

[15] J. Zhu, et al., "Physical-Layer Security and Reliability Challenges for Industrial Wireless Sensor Networks," IEEE Access, vol. 5, pp. 5313-5320, 2017.

[16] D. Fernandes, et al., "A Low Traffic Overhead Transmission Power Control for Wireless Body Area Networks," IEEE Sensors Journal, vol. 18, pp. 1301-1313, 2018

[17] K. Kang, et al., "Game-Theory-Based Distributed Power Splitting for Future Wireless Powered MTC Networks," IEEE Access, vol. 5, pp. 20124-20134, 2017.

[18] H. Feng and J. Dong, "Reliability analysis for WSN based on a modular k-out-of-n system," Journal of Systems Engineering and Electronics, vol. 28, pp. 407-412, 2017. 
[19] X. Liu, et al., "Power allocation for energy harvesting in Wireless Body Area Networks," China Communications, vol. 14, pp. 22-31, 2017.

[20] R. Prabha, et al., "Energy Efficient Data Acquisition Techniques Using Context Aware Sensing for Landslide Monitoring Systems," IEEE Sensors Journal, vol. 17, pp. 6006-6018, 2017.

[21] A. Samanta and S. Misra, "Energy-Efficient and Distributed Network Management Cost Minimization in Opportunistic Wireless Body Area Networks," IEEE Transactions on Mobile Computing, vol. 17, pp. 376-389, 2018.

[22] C. Wang, et al., "Combining Solar Energy Harvesting with Wireless Charging for Hybrid Wireless Sensor Networks," IEEE Transactions on Mobile Computing, vol. 17, pp. 560-576, 2018.

[23] T. Wang, et al., "Fog-Based Evaluation Approach for Trustworthy Communication in Sensor-Cloud System," IEEE Communications Letters, vol. 21, pp. 2532-2535, 2017.

[24] A. E. Zonouz, et al., "Hybrid wireless sensor networks: A reliability, cost and energy-aware approach," IET Wireless Sensor Systems, vol. 6, pp. 42-48, 2016.

[25] A. Wolf, et al., "Asymptotically Optimal Power Allocation for WSNs with Mutually Correlated Sensing Data," IEEE Communications Letters, vol. 20, pp. 1317-1320, 2016.

[26] M. K. Naeem, et al., "Universal and Dynamic Clustering Scheme for Energy Constrained Cooperative Wireless Sensor Networks," IEEE Access, vol. 5, pp. 12318-12337, 2017.

[27] L. Lei, et al., "Optimal Reliability in Energy Harvesting Industrial Wireless Sensor Networks," IEEE Transactions on Wireless Communications, vol. 15, pp. 5399-5413, 2016.

[28] G. Liu, et al., "QoS-Aware Throughput Maximization in Wireless Powered Underground Sensor Networks," IEEE Transactions on Communications, vol. 64, pp. 4776-4789, 2016.

[29] D. Wu, et al., "An Energy-Efficient Data Forwarding Strategy for Heterogeneous WBANs," IEEE Access, vol. 4, pp. 7251-7261, 2016.

[30] G. Campobello, et al., "Improving Energy Saving and Reliability in Wireless Sensor Networks Using a Simple CRT-Based Packet-Forwarding Solution,” IEEE/ACM Transactions on Networking, vol. 20, pp. 191-205, 2012.

[31] Raad Farhood Chisab, "An Active Technique for Power Saving in WSN under Additive White Gaussian Noise Channel," International Journal of Electrical and Computer Engineering, vol. 9, 2017.

[32] Manjunath and Rao, "Balancing Trade-off between Data Security and Energy Model for Wireless Sensor Network," International Journal of Electrical and Computer Engineering, vol. 8, 2017.

[33] Jayashri B. S. and G. R. Rao, "FRDF: Framework for Reliable Data Fusion to Leverage Communication Performance in Sensor Network," Springer-Software Engineering Trends and techniques in Intelligent Systems, Advances in Intelligent Systems and Computing, 2017.

[34] Jayashri B. S. and G. R. Rao, "EEDF: Energy Efficient Data Fusion Supportive of Virtual Multipath Propagation in WSN," International Journal of Applied Engineering Research, vol. 10, pp. 54-59, 2015.

[35] Jayashri B. S. and G. R. Rao, "RaESS: Reliable-and-Efficient Statistical Spreading Data Fusion Mechanism in Wireless Sensor Network," Springer-Computer Science On-line Conference, Software Engineering Trends and techniques in INtelligent Systems, 2017.

[36] H. Luo, et al., "Data fusion with desired reliability in wireless sensor networks," IEEE Transactions on Parallel and Distributed Systems, vol. 22, pp. 501-513, 2011.

[37] K. Zhang, et al., "Wireless sensor data fusion algorithm based on the sensor scheduling and batch estimate," International Journal of Future Computer and Communication, vol. 2, pp. 333, 2013.

\section{BIOGRAPHIES OF AUTHORS}

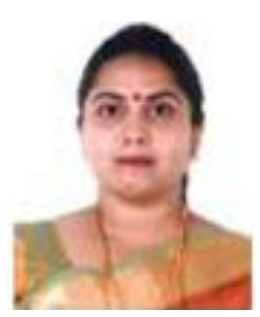

Jayasri B. S. is currently working as an Associate Professor in the department of CS\&E, The National Institutional of Engineering, and Mysuru. She obtained her M.tech degree in (2006) from Visvesvaraya Technical University, Belgaum. She is currently pursuing her Ph.D., and her area of interest includes Wireless Sensor Networks, Wireless Communication, Computer Networks, Operational Research and System Simulation Modeling.

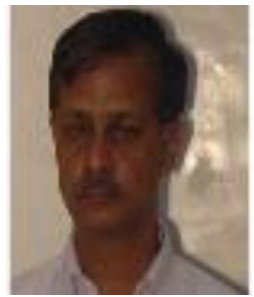

G. Raghavendra Rao took his BE ME and PhD degrees from UOM, IISC, Bangalore and UOM respectively. After serving as Asst. Engineer (Jaguar Project) at HAL, Bangalore for 4 years, he joined NIE as a faculty in June 1983. He was the first faculty of the department of Computer Science \& Engg., and was responsible for the establishment of the department, facilities and Laboratories of CS, IS and MCA departments in NIE. He became Asst. Professor in January 1990 and Professor of CSE in January 1995 respectively. He was Principal (I/C) of NIE from March 2004 to April 2008. He was also the founder Principal of NIE Institute of Technology from July 2008 to October 2010. 